\title{
Elevação da razão normalizada internacional por interação medicamentosa em paciente crítico de unidade de terapia intensiva: relato de caso
}

\section{Elevation of the International Normalized Ratio for drug interaction in critically ill intensive care unit patients: report of a clinical case}

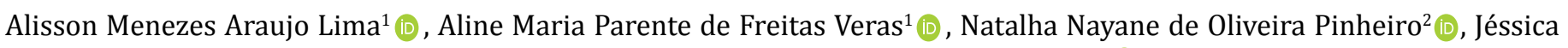
Bezerra da $\operatorname{Costa}^{2}$ (D) , Sandna Larissa Freitas dos $\operatorname{Santos}^{2}$ (D), Athila Wesley Lima Lacerda² (D)

1. Farmácia Clínica da Maternidade Escola Assis Chateaubriand, Fortaleza, CE, Brasil. 2. Residência Multiprofissional em Atenção Hospitalar à Saúde pela Maternidade Escola Assis Chateaubriand (MEAC), Fortaleza, CE, Brasil.

\section{Resumo}

Relato de caso: o caso relatado apresenta uma paciente com uso de anticoagulantes e antimicrobianos que resultou na elevação dos valores do parâmetro de tempo de protombina e razão normalizada internacional, associados às condições hemodinâmicas. Conclusão: a interação medicamentosa foi identificada por um farmacêutico clínico e comunicada à equipe médica, que alterou a prescrição, observando-se melhora nos exames.

Palavras-chave: Unidades de Terapia Intensiva. Transtornos da Coagulação Sanguínea. Serviço de Farmácia Hospitalar.

\begin{abstract}
Case report: the case report shows a patient with anticoagulants and antimicrobials that resulted in the elevation of the values of the prothrombin time parameters and INR, associated with hemodynamic conditions. Conclusion: the drug interaction was identified by the clinical pharmacist; the medical team changed the prescription, and an improvement in the exam results was observed.
\end{abstract}

Key words: Intensive Care Units. Blood Coagulation Disorders. Pharmacy Service, Hospital.

\section{INTRODUÇÃO}

Paciente em estado clínico crítico é aquele que apresenta comorbidades de um ou mais órgãos vitais, com instabilidade hemodinâmica, necessitando de cuidados intensos e controle vital com maior frequência. Além dos riscos relacionados à complexidade da terapia, que pode ser administrada por meio de caráter invasivo, é comum a ocorrência de eventos que podem piorar as condições clínicas desses pacientes ${ }^{1}$.

Os eventos tromboembólicos estão entre as principais causas de mortes evitáveis em pacientes hospitalizados, sendo o pós-operatório um importante fator de risco. Pensando na condição clínica de um paciente crítico, percebe-se a importância da prevenção de tromboembolismo venoso (TEV) e tromboembolismo pulmonar (TEP), a qual deve ser feita com medicamentos anticoagulantes. Além disso, é de extrema necessidade o monitoramento dos níveis de anticoagulação².

O Tempo de Protrombina ou Tempo de Atividade da Protrombina (TAP) e a Razão Normalizada Internacional (INR) são as medidas laboratoriais utilizadas para avaliar a via extrínseca da coagulação ${ }^{3}$. O principal objetivo é a prevenção de eventos tromboembólicos, como Trombose Venosa Profunda (TVP), com o menor risco hemorrágico. A metodologia do teste, por meio da mensuração do INR e a análise laboratorial com coagulômetros de amostra do sangue venoso e o nível adequado de INR para uma anticoagulação eficaz e segura, em diversas indicações, está no intervalo de 2,0 a 3,04.

Mesmo com o advento dos novos anticoagulantes orais, a Comissão Nacional de Incorporação de Tecnologias no SUS (CONITEC), ao avaliar e comparar com os tratamentos anticoagulantes já preconizados, relatou que as evidências atualmente disponíveis sobre eficácia e segurança dos novos anticoagulantes orais (apixabana, dabigatrana e rivaroxabana) para prevenção de AVC isquêmico são baseadas em ensaios clínicos fase III de não inferioridade. Não foram localizadas, até o momento, evidências estatisticamente significativas para apixabana e rivaroxabana em relação ao desfecho prevenção de AVC isquêmico. As vantagens oferecidas pelos novos 
anticoagulantes incluem a conveniência de não necessitar de testagem rotineira da coagulação (INR) e a ausência de interações com alimentos. Entre as desvantagens, além de seus maiores custos, destacam-se a impossibilidade de uso em pacientes com insuficiência renal grave, o uso em duas doses diárias, a impossibilidade de controlar seu efeito por testes laboratoriais e a ausência de antídoto em alguns casos. Em relação à dabigatrana, os efeitos gastrointestinais impossibilitam o uso em uma parcela dos pacientes 5 .

\section{RELATO DO CASO}

Paciente do sexo feminino, 41 anos, com histórico de seis gestações, sendo o desfecho de umas delas o aborto, foi admitida na UTI em pós-operatório de curetagem por abortamento infectado, tendo seu caso evoluído para Insuficiência Renal Aguda Dialítica, Insuficiência Respiratória Aguda, Hepatopatia isquêmica e TVP em Membro Superior Direito. Após 29 dias de internação na UTI, a paciente foi transferida para enfermaria.

Baseado no quadro clínico na UTI, foram prescritos antimicrobianos e terapia anticoagulante com varfarina e bomba de infusão de heparina sódica. No primeiro dia de internação na enfermaria, devido ao quadro da paciente, a equipe médica optou por adicionar à terapia antimicrobiana fluconazol e levofloxacina. Os exames laboratoriais de coagulação foram analisados e encontravam-se sem alterações. Além disso, a paciente estava em uso dos medicamentos Polimixina B, Amicacina, Teicoplanina e Omeprazol para condicionamento de seu quadro clínico.

No entanto, após o uso concomitante, por três dias, dos anticoagulantes, fluconazol e levofloxacino, indicados para tratamento de sete dias, a paciente queixou-se de aperto no peito e parestesia em região oral e membro superior direito. Nesse período, os exames de coagulação começaram a apresentar alterações significativas, Tempo de Protrombina $=$ 67,2 segundos e Razão Normalizada Internacional = 5,6 (INR) e, dessa forma, houve a introdução da vitamina $k$, e a heparina e a varfarina foram suspensas para a avaliação dos exames de coagulação, retornando à prescrição dois dias depois em concentrações reduzidas. Entretanto, os resultados do INR continuaram elevados e, no decorrer dos dias, o valor do INR chegou a ficar maior que 14 (gráfico 1). Não houve alteração da via de administração dos medicamentos até a transferência da paciente.

Gráfico 1. Alargamento do INR devido à interação medicamentosa decorrente do uso concomitante de fluconazol, levofloxacina e varfarina.

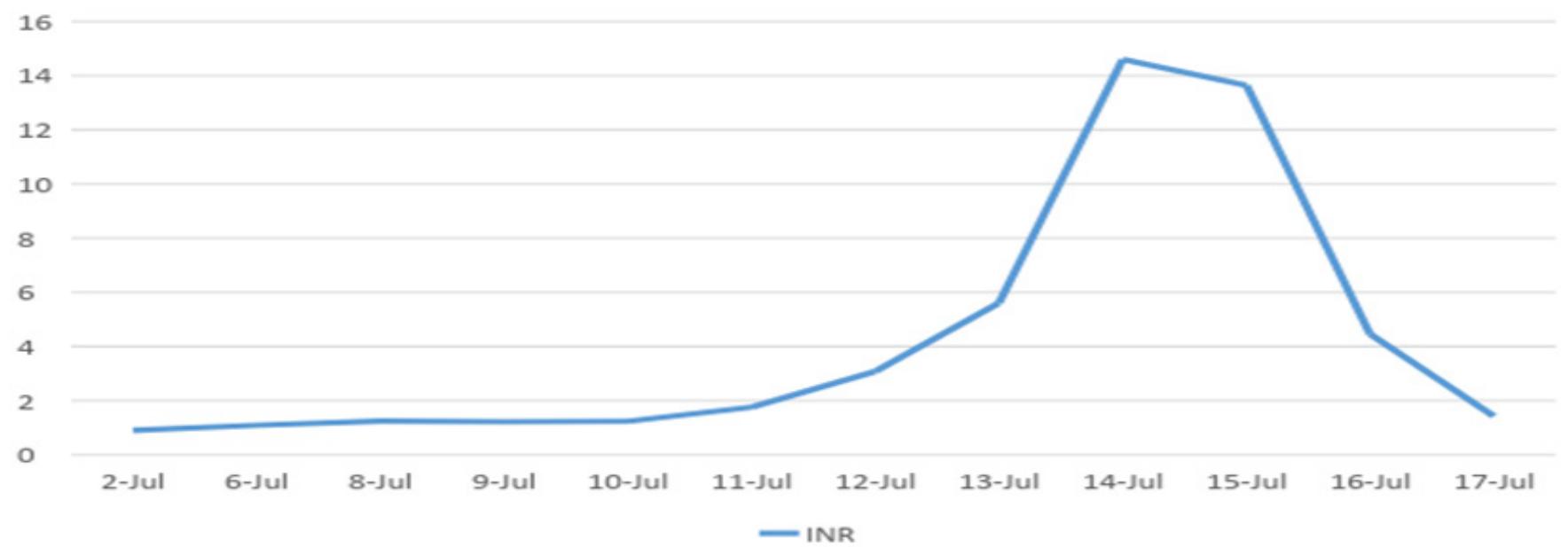

Legenda: 1- dia 10 jul: início dos antimicrobianos já em uso de anticoagulantes; 2: dia 14 jul - suspensão do anticoagulante; 3 : dia 16 jul suspensão dos antimicrobianos.

Diante do caso, a equipe multiprofissional se reuniu para discutir, momento em que o farmacêutico clínico analisou a prescrição médica e observou o uso simultâneo dos medicamentos mencionados, sendo feita a verificação das interações entre eles, tendo como principal efeito o alargamento do INR. A base de dados utilizada para verificação de interações medicamentosas foi o MICROMEDEX ${ }^{6}$, que classificou as interações como graves (no quesito gravidade) e excelente (no quesito documentação), trazendo um ótimo respaldo para intervenção farmacêutica.

O caso foi discutido pela equipe multiprofissional composta por médico, enfermeiro, farmacêutico e nutricionista, ocasião em que a relação risco-benefício da paciente foi avaliada por todos os profissionais. Considerando a relevância da interação classificada como grave, a conduta tomada foi a suspenção da terapia anticoagulante, a revisão dos exames periodicamente e o término dos antimicrobianos, uma vez que a sintomatologia da infecção se encontrava resolvida. Após a suspensão dos medicamentos, os exames de coagulação começaram a normalizar-se. Não foi verificada nenhuma causalidade da ocorrência do evento com os outros medicamentos utilizados; além disso, não houve realização de antibiograma, não sendo possível evidenciar a sensibilidade do espectro necessário para a terapia da infecção. 
Para classificar causalidade do evento, foi utilizado o Algoritmo de Naranjo ${ }^{7}$ que tem a finalidade de buscar informações sobre a reação adversa ao medicamento, apresentando uma escala de probabilidade que inclui a sequência cronológica entre a administração do medicamento suspeito e o surgimento de manifestações clínicas, a plausibilidade da relação de causalidade (levando em consideração a descrição prévia da reação na literatura médica ou as propriedades farmacológicas conhecidas do medicamento). A ferramenta apresenta uma pontuação de acordo com as informações em que maior ou igual a 9 é considerada definida; entre 5 e 8 é provável; entre 1 e 4 é possível; e menor ou igual a 0 é apresentada como duvidosa. De acordo com os dados obtidos e analisados, constatou que o evento atingiu pontuação 6 e foi classificado com provável.

\section{DISCUSSÃO}

A terapia de escolha para tratamento da TVP na paciente foi a com heparina não fracionada, por via endovenosa, associada à varfarina, anticoagulante oral de escolha para o tratamento e a prevenção de complicações trombóticas ${ }^{8}$. A varfarina atua inibindo a síntese da vitamina $\mathrm{k}$ e dos fatores de coagulação no fígado, além de diminuir a produção de proteínas anticoagulantes. É predominantemente eliminada pelo fígado tendo um metabolismo dependente do citocromo P450A. Dessa forma, esse medicamento possui o risco de sangramento como principal reação adversa, devido ao alargamento do INR e sua metabolização hepática ${ }^{9}$.

Foi verificado que ambos os antimicrobianos, quando associados ao anticoagulante oral, podem aumentar o risco de sangramento. De acordo com o Micromedex6, fluconazol, como um inibidor do CYP3A4, aumenta as concentrações plasmáticas da varfarina, um substrato do CYP3A4, e consequente aumento do INR e do TAP, podendo elevar o risco de hemorragia.

Schelleman et $\mathrm{al}^{10}$. mostraram, em sua pesquisa de póscomercialização, relatos de pacientes que receberam fluconazol com varfarina da ocorrência de hematomas, epistaxe, sangramento gastrointestinal, hematúria e melena. No estudo de caso-controle realizado por Baillargeon et al ${ }^{11}$, com usuários contínuos de varfarina com 65 anos ou mais, houve um aumento de 4,5 vezes no risco de sangramento, requerendo hospitalização com exposição a antifúngicos azólicos, incluindo o fluconazol.

Outro estudo de caso-controle apresentou um aumento de $48 \%$ do risco de sangramento, requerendo hospitalização com exposição a qualquer terapia com antibióticos ${ }^{4}$. Estudos mostram que o mecanismo da interação de levofloxacina e varfarina sugere a alteração na flora intestinal que sintetiza a vitamina $\mathrm{K}^{12}$. Baillargeon et al ${ }^{11}$, nessas situações, sugerem a substituição de levofloxacina por um antibiótico com um perfil de baixo risco para sangramento, como clindamicina e cefalexina. Quando não for possível, devem-se monitorar aumentos no INR e tempo de protrombina.

A percepção da ocorrência de interações medicamentosas em pacientes críticos deve ser adotada pela equipe multiprofissional; porém, o farmacêutico tem habilidade de identificação e manejo clínico no acompanhamento frequente e individualizado no uso múltiplo de medicamentos associados a condições desfavoráveis dos pacientes em UTI com vistas em uso de outro antimicrobiano com o mesmo espectro da quinolona, acompanhamento da evolução clínica da paciente, monitoramento dos exames laboratoriais e vigilância de erros de medicação ${ }^{11}$. Além disso, constata-se a necessidade de melhoria no sistema de distribuição de medicamentos da instituição, como estratégia de monitoramento e eventual barreira de falhas dos eventos negativos que, por meio de uma análise criteriosa da prescrição e monitoramento efetivo, preveniria a interação e os possíveis danos à paciente ${ }^{13}$.

Levando-se em consideração os altos riscos para erros de medicamentos e reações adversas a medicamentos, devido à natureza crítica de suas doenças, à polifarmácia, à utilização de medicamentos de alto risco e a uma frequência alta de mudanças na farmacoterapia, o farmacêutico é capacitado a contribuir para o cuidado do paciente por revisar e fazer recomendações, racionalizar a terapia medicamentosa, com o objetivo de maximizar a segurança e os resultados terapêuticos ${ }^{14}$.

Este relato de caso foi aprovado pelo Comitê de Ética e Pesquisa da instituição com o número do Parecer: 3.046.226.

\section{REFERÊNCIAS}

1. Safavi K, Wiener-Kronish J, Hanidziar D. The Complexity and Challenges of Intensive Care Unit Admissions and Discharges: Similarities With All Hospitalized Patients. JAMA Intern Med. 2018 Oct; 178(10): 1399-1400. doi: 10.1001/ jamainternmed.2018.3674

2. Vitor SKS, Daou JP, Góis AFT. Prevenção de tromboembolismo venoso (trombose venosa profunda e embolia pulmonar) em pacientes clínicos e cirúrgicos. Diagn. Tratamento. 2016; 21(2): 59-64.

3. Flato UAP, Buhatem T, Merluzzi T, Bianco ACM. Novos anticoagulantes em cuidados intensivos. Rev. Bras. ter. intensiva. 2011; 23(1): 68-77. doi: http:// dx.doi.org/10.1590/S0103-507X2011000100012.

4. Araújo ACO, Domingues RB, Bellen BV. Determinação do INR: comparação entre método convencional e dispositivo portátil. J Vasc Bras. 2014 Abr-Jun;
13(2):88-93. doi: http://dx.doi.org/10.1590/jvb.2014.051.

5. CONITEC. Comissão Nacional de Incorporação de Tecnologias no SUS. Consulta Pública de número 82. Disponível em: http://conitec.gov.br/images/ Relatorios/2016/Relatrio_Anticoagulantes_final.pdf Acessado em 03/05/2019.

6. Micromedex 2.0 [Internet]. Ann Arbor (MI): Truven Health Analytics Inc. 2013. Fluconazole, levofloxacin and warfarin.; [citado em 2018 Ago 22]. Disponível em: https://www.micromedexsolutions.com/home/dispatch.

7. Naranjo CA, Busto U, Sellers EM, Sandor P, Ruiz I, Roberts EA, et al. Method for estimating the probability of adverse drug reactions. Clin. Pharmacol. Ther., 1981; 30(2): 239-245. doi: 10.1038/clpt.1981.154.

8. Sociedade Brasileira de Angiologia e de Cirurgia Vascular. Trombose Venosa 
Profunda: Diagnóstico e Tratamento. São Paulo: SBACV; 2011.

9. Qmar A, Vaduganathan M, Greenberger NJ Giugliao RP. Oral Anticoagulation in Patients With Liver Disease. J Am Coll Cardiol. 2018 May 15; 71(19): 2162-75. doi: doi: 10.1016/j.jacc.2018.03.023.

10. Schelleman H, Bilker WB, Brensinger CM, Han X, Kimmel SE, Hennessy S. Warfarin with fluoroquinolones, sulfonamides, or azole antifungals: interactions and the risk of hospitalization for gastrointestinal bleeding. Clin Pharmacol Ther. 2008 Nov; 84(5):581-588. doi: 10.1038/clpt.2008.150.

11. Baillargeon J, Holmes HM, Lin YL Raji MA, Sharma G, Kuo YF. Concurrent use of warfarin and antibiotics and the risk of bleeding in older adults. Am J Med Feb, 2012; 125(2):183-189. doi: 10.1016/j.amjmed.2011.08.014.

12. Lane MA: Serious bleeding events due to warfarin and antibiotic coprescription in a cohort of veterans. Am J Med Jul, 2014; 127(7):657-663.e2 doi: 10.1016/j.amjmed.2014.01.044.

13. Saraiva DMRF. Erros de Medicação: tipos, taxonomia, impacto, causalidade e estratégias de gestão do risco. Egitania Sciencia. 2016 Jul-Dec: 7-22.

14. Medeiros RA, Moraes JP. Intervenções farmacêuticas em prescrições médicas na Unidade de Terapia Intensiva. Rev. Bras. Farm. Hosp. Serv. Saúde São Paulo. 2014 Jun; 5(2): 26-29.

\section{Como citar este artigo/How to cite this article:}

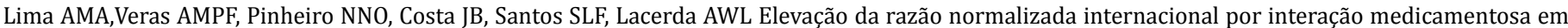
paciente crítico de unidade de terapia intensiva: relato de caso. J Health Biol Sci. 2019 Out-Dez; 7(4):436-439. 